\title{
Automated real-time detection of tonic-clonic seizures using a wearable EMG device
}

Sándor Beniczky, MD, PhD, Isa Conradsen, PhD, Oliver Henning, MD, Martin Fabricius, MD, DMSc, and Peter Wolf, MD, DMSc

Neurology ${ }^{\circledR}$ 2018;90:e428-e434. doi:10.1212/WNL.0000000000004893

\section{Abstract}

\section{Objective}

To determine the accuracy of automated detection of generalized tonic-clonic seizures (GTCS) using a wearable surface EMG device.

\section{Methods}

We prospectively tested the technical performance and diagnostic accuracy of real-time seizure detection using a wearable surface EMG device. The seizure detection algorithm and the cutoff values were prespecified. A total of 71 patients, referred to long-term video-EEG monitoring, on suspicion of GTCS, were recruited in 3 centers. Seizure detection was real-time and fully automated. The reference standard was the evaluation of video-EEG recordings by trained experts, who were blinded to data from the device. Reading the seizure logs from the device was done blinded to all other data.

\section{Results}

The mean recording time per patient was 53.18 hours. Total recording time was 3735.5 hours, and device deficiency time was 193 hours (4.9\% of the total time the device was turned on). No adverse events occurred. The sensitivity of the wearable device was $93.8 \%$ (30 out of 32 GTCS were detected). Median seizure detection latency was 9 seconds (range -4 to 48 seconds). False alarm rate was $0.67 / \mathrm{d}$.

\section{Conclusions}

The performance of the wearable EMG device fulfilled the requirements of patients: it detected GTCS with a sensitivity exceeding $90 \%$ and detection latency within 30 seconds.

\section{Classification of evidence}

This study provides Class II evidence that for people with a history of GTCS, a wearable EMG device accurately detects GTCS (sensitivity $93.8 \%$, false alarm rate $0.67 / \mathrm{d}$ ).

\author{
Correspondence \\ Dr. Beniczky \\ sbz@filadelfia.dk
}

\section{- CME Course \\ NPub.org/cmelist}

RELATED ARTICLE

Editorial

Non-EEG seizure detection is here

Page 207

MORE ONLINE

$\rightarrow$ Class of evidence

Criteria for rating

therapeutic and diagnostic studies

NPub.org/coe 


\section{Glossary}

$\mathbf{C I}=$ confidence interval; EDDI = Epileptic seizure Detector Developed by IctalCare; EMU = epilepsy monitoring unit; FAR = false alarm rate; GTCS = generalized tonic-clonic seizures; STARD = Standards for Reporting of Diagnostic Accuracy; SUDEP = sudden unexpected death in epilepsy.

Generalized tonic-clonic seizures (GTCS) may lead to injuries, and constitute the major risk factor for sudden unexpected death in epilepsy (SUDEP), especially in unattended patients. ${ }^{1,2}$ Wearable seizure detection devices were suggested to prevent SUDEP. ${ }^{1-4}$ The unpredictability of seizure occurrence is distressing for patients and caregivers. ${ }^{3}$ It contributes to social isolation and decreased quality of life, and surveys showed a need for wearable seizure detection devices. ${ }^{5-10}$

Therapeutic decisions and clinical trials use self-reporting of seizures. However, this is unreliable: studies in video-EEG monitoring units demonstrated that $61 \%$ of seizures remain unrecognized by patients, even secondarily generalized tonicclonic seizures, which remained completely unrecognized. ${ }^{11}$ Wearable devices could provide objective data about seizure occurrence.

Signals from muscles, recorded with surface EMG, seem to be a promising modality for detecting motor seizures. A recently published review summarizes the specific pathophysiologic changes in muscle activity during seizures. ${ }^{12}$ Quantitative EMG parameters differentiate epileptic from physiologic muscle activation and from nonepileptic seizures. ${ }^{13-15}$ Based on characteristic changes in frequency and amplitude, ${ }^{16}$ we have developed a generic seizure detection algorithm. ${ }^{17}$ High sensitivity and specificity of EMG-based seizure detection algorithms were found in 2 retrospective studies using offline analysis of EMG recorded with conventional amplifiers. ${ }^{17,18} \mathrm{~A}$ pilot study showed promising results for a wearable EMGbased device implementing our algorithm. ${ }^{19}$

We present a prospective, multicenter study on the diagnostic accuracy of real-time detection of GTCS using the wearable EMG device. We report our findings according to the Standards for Reporting of Diagnostic Accuracy (STARD) criteria. ${ }^{20}$

\section{Methods}

\section{Patients}

Seventy-one consecutive patients ( 32 female; age 10-62 years, mean 34.1 years, median 34 years) were recruited to the study: 33 patients at the Danish Epilepsy Centre, Dianalund; 5 patients at Copenhagen University Hospital Rigshospitalet, Denmark; and 33 patients at the National Centre for Epilepsy, Oslo University Hospital, Norway, between October 21, 2014, and January 23, 2017. The median baseline monthly frequency of GTCS according to the patients' seizure logs was 1.75 .
Inclusion criteria were (1) admission to noninvasive longterm video-EEG monitoring in the epilepsy monitoring unit (EMU) in one of the participating centers; and (2) historical data suggesting that the patient had GTCS. Exclusion criteria were (1) age under 3 years; (2) pregnancy; (3) allergy to the self-adhesive patch; or (4) implanted vagal nerve stimulator or deep brain stimulator.

Patients underwent video-EEG monitoring using wireless amplifiers (at the Danish Epilepsy Centre) or connected with a 10-meter-long cable (in the other centers). The mobility of the patients was not restricted in the EMUs, where patients were free to perform their habitual physical activities and participate in activities such as using an exercise bicycle, preparing coffee or food, and playing home video games involving body motion (Nintendo [Kyoto, Japan] Wii). ${ }^{21}$ Patients were under continuous surveillance and no injuries occurred during monitoring.

\section{Standard protocol approvals, registrations, and patient consent}

The project has been approved by the regional ethics committees (in Denmark: SJ-380; in Norway: 2013/2354). All patients (or guardians of patients) gave their written, informed consent prior to the study.

\section{Wearable EMG-based seizure detection device}

We tested the diagnostic accuracy of the Epileptic seizure Detector Developed by IctalCare (EDDI). The device measures surface EMG signals and was placed on the brachial biceps muscle (figure 1, A-D). The device is relatively small, and is easily hidden under the patient's clothes. The choice of side for the placement was decided based on historical data where the patient's seizures habitually were most strongly expressed. When there was no known side preponderance, the device was placed on the left.

The wearable device is attached to a self-adhesive hypoallergenic hydrogel patch (Axelgaard Manufacturing Co. Ltd., Fallbrook, CA) with 3 embedded surface EMG electrodes (bipolar recording electrodes and ground electrode), developed specifically for this device (figure $1 \mathrm{C}$ ). The distance between the recording electrodes was $20 \mathrm{~mm}$. A lithium polymer battery was placed into the wearable device.

Sampling frequency was $1,024 \mathrm{~Hz}$. A modified version of the previously reported algorithm was implemented into the device. ${ }^{17,19}$ Briefly, the electrophysiologic biomarker of the initial phase of GTCS consists of an increase in amplitude and high-frequency $(>150 \mathrm{~Hz})$ oscillations in the EMG signal. 


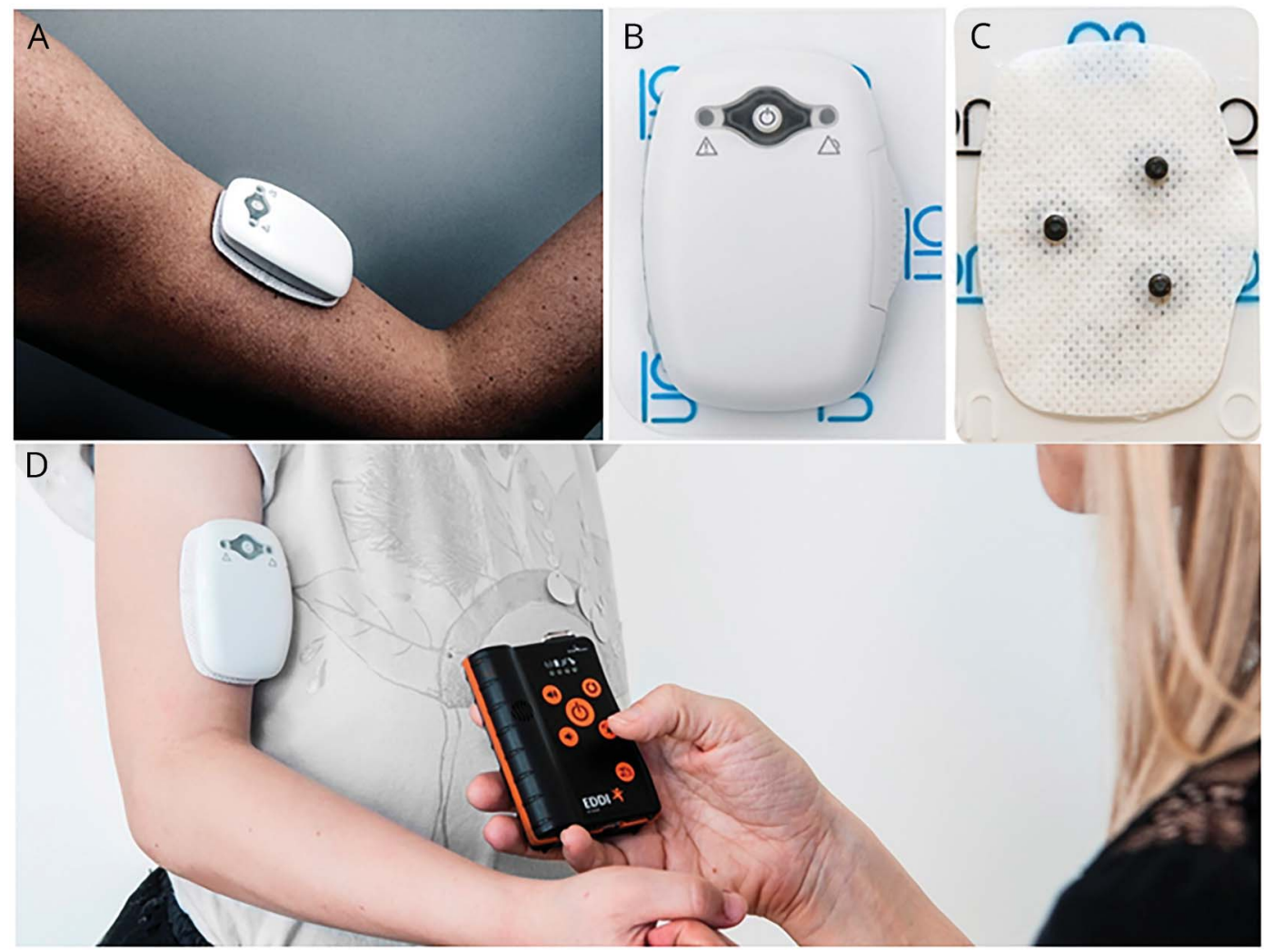

(A) The wearable device placed on the brachial biceps muscles. $(B, C)$ The wearable device, which is connected to the self-adhesive patch, containing the recording electrodes and the ground electrode. (D) Remote control of the device.

This was expressed as the number of zero-crossings, where high frequency oscillations (high-pass filtered at $150 \mathrm{~Hz}$ ) exceeded a hysteresis of $\pm 35 \mu \mathrm{V}$. The count of zero-crossings was calculated for a window of 1 second, with an overlap of $75 \%$ with the previous window. When this exceeded the predefined threshold, the alarm was triggered (figure 2). The measures are generic (not patient-specific), they were prespecified before this study, and they were applied to all patients. The algorithm requires minimal computational power.

The device gives real-time seizure alarms and a different type of alarm for device deficiency, when electrode impedance is out of bounds. However, the study design implied blinding of the personnel for any data from the device, and therefore these alarms were disabled in this study. Instead, the device logged real-time all-seizure alarm time points, for comparison with the reference standard, and the periods with high impedance and $50-\mathrm{Hz}$ artefacts were considered device deficiency periods. Detected seizure time points were read blinded to any other data. In addition, the EMG recorded by EDDI was transmitted via radio signals to a computer that stored the signals. The intention was to use these signals for further optimization and fine-tuning the measures of the algorithm. However, that is not reported in this study. All data presented in this study are based on real-time seizure detection using the predefined measures and the algorithm described above, with all calculations done on the wearable device.

The self-adhesive patch was changed once per day (standard procedure). The battery was changed 3 times per day; this is more than the standard procedure for the device, and it was necessary because in the study EMG data were transmitted to and saved on a computer. This increased significantly the power consumption of the device. For standard operation of EDDI (real-time computation, giving alarms and logging time points, without transmitting EMG data), change of the battery is required once per day.

\section{Reference standard}

The reference standard for identifying seizures was long-term video-EEG monitoring, reviewed by trained, board-certified clinical neurophysiologists and epileptologists, with more than 10 years' experience with EMU (S.B., O.H., and M.F.). The experts were blinded to all data from EDDI, until they completed the analysis of the video-EEG recordings. For GTCS, they identified the start time point at the beginning of the tonic phase and the end time point at the end of the clonic phase. The time points of the reference standard were then compared with the logs of the real-time automated seizure 
Figure 2 EMG-based seizure detection algorithm

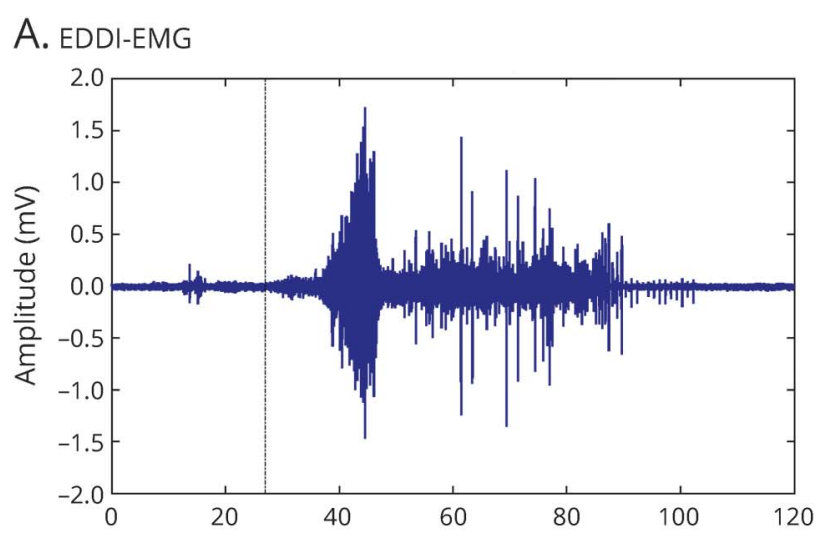

B. Zero-crossings

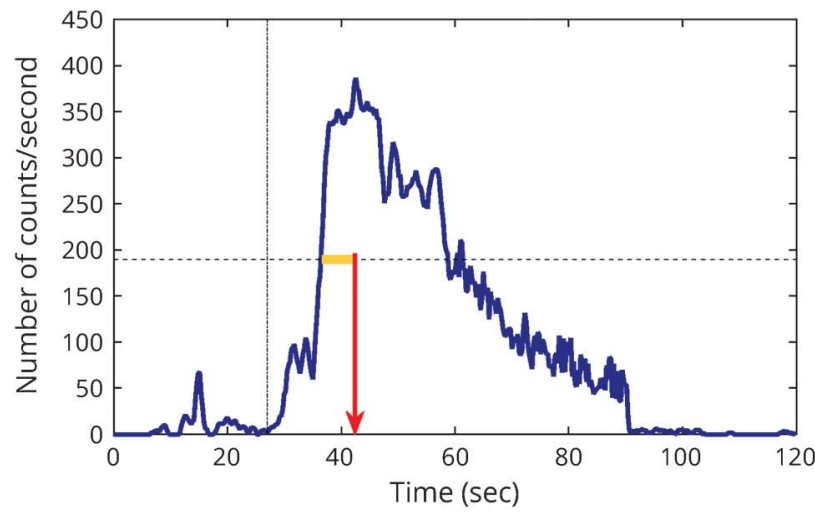

(A) The EMG signals recorded by Epileptic seizure Detector Developed by IctalCare (EDDI) during a generalized tonic-clonic seizure (high-pass filter: $150 \mathrm{~Hz}$ ). (B) The number of zero-crossings calculated from the signal in (A). The horizontal axis in both $(A)$ and $(B)$ is the time (in seconds). When the number of zero-crossings exceeds the threshold (dotted horizontal line) for the prespecified time window (yellow line), then the seizure alarm is triggered (red vertical arrow).

detection. At the beginning of each monitoring, the times in the device and in the video-EEG monitoring equipment were synchronized.

\section{Study outcome measures}

The primary aim was determining the diagnostic accuracy of the device. Sensitivity was expressed as the percentage of the detected GTCS. Specificity was expressed as the false alarm rate (FAR), calculated from the entire patient population.

In addition, we documented the following: adverse events (any patient concern related to the device, reported by the patient or observed by the personnel), device-off time (total time when the device was turned off during monitoring-for example, for changing patch and battery, time in bathroom, ictal SPECT), device-deficiency time (periods with high impedance and artefacts preventing the functioning of the device; this was expressed as percentage of the total time when the device was turned on-i.e., the total recording time), and detection latency (measured from the start time point identified by the experts to the alarm time point from the device).

\section{Classification of evidence}

The primary research question was the accuracy (sensitivity and false-alarm rate) of a wearable EMG device for detecting GTCS. This study provides Class II evidence that for people with a history of GTCS, the wearable EMG device, EDDI, detects GTCS with a sensitivity of $93.8 \%$ and a false-alarm rate of $0.67 / \mathrm{d}$.

\section{Sample size estimation}

For the expected sensitivity of $95 \%^{17,19}$ and expected prevalence of $28 \%$ in the studied population, ${ }^{22}$ with a confidence interval (CI) of $10 \%$, we needed 18 patients with GTCS and 65 recruited patients. ${ }^{23}$ GTCS is the seizure type with the highest risk for the patients and therefore considered an undesired event in EMUs. ${ }^{24}$ Diagnostic tapering of antiepileptic medication is aimed at increasing seizure frequency though avoiding GTCS unless this is the targeted seizure type, and these occur relatively rarely in EMUs. Our intention was to record GTCS from 20 patients, with a total of at least 30 GTCS. Since the quantitative EMG changes during GTCS have a high intraindividual and interindividual consistency, this number seemed to be sufficient to evaluate the performance of the device. ${ }^{14}$

\section{Results}

The mean recording time per patient was 53.18 hours (median 53.12; range 2.3-130 hours). The device was turned on for 3,928.6 hours. Device deficiency occurred in 193.1 hours (4.9\%). The total recording time was $3,735.5$ hours. No adverse events occurred.

Figure 3 shows the STARD flowchart of the study. Thirty-two GTCS occurred during the recording time. Twenty of the 71 included patients had GTCS (11 patients had 1 seizure each; 6 patients had 2 seizures; 3 patients had 3 seizures).

The sensitivity of the device was $93.8 \%$ as it detected 30 out of the 32 GTCS (95\% CI 86\%-100\%). All GTCS were detected in 18 patients ( $90 \%$ of all patients with GTCS). Two patients with 2 seizures had only 1 seizure detected. Calculated per patient, the mean sensitivity was $95 \%$ (median 100\%; range 50\%-100\%). Four seizures from 3 patients with idiopathic generalized epilepsy were primary GTCS; all of them were detected. The other seizures were secondarily GTCS.

Both missed GTCS occurred as the second seizure after a detected GTCS, with short interseizure periods (19 minutes and 98 minutes, respectively). Retrospective inspection of the EMG signals during the missed seizures showed low amplitude of the EMG signal in one case, and very short tonic phase in the other case, so that the number of zero-crossings did not exceed the predefined detection threshold for any of the missed seizures.

The median detection latency of the device was 9 seconds (range -4 to 48 seconds; mean 12.3 seconds), measured from the start of the tonic phase of the seizures. 


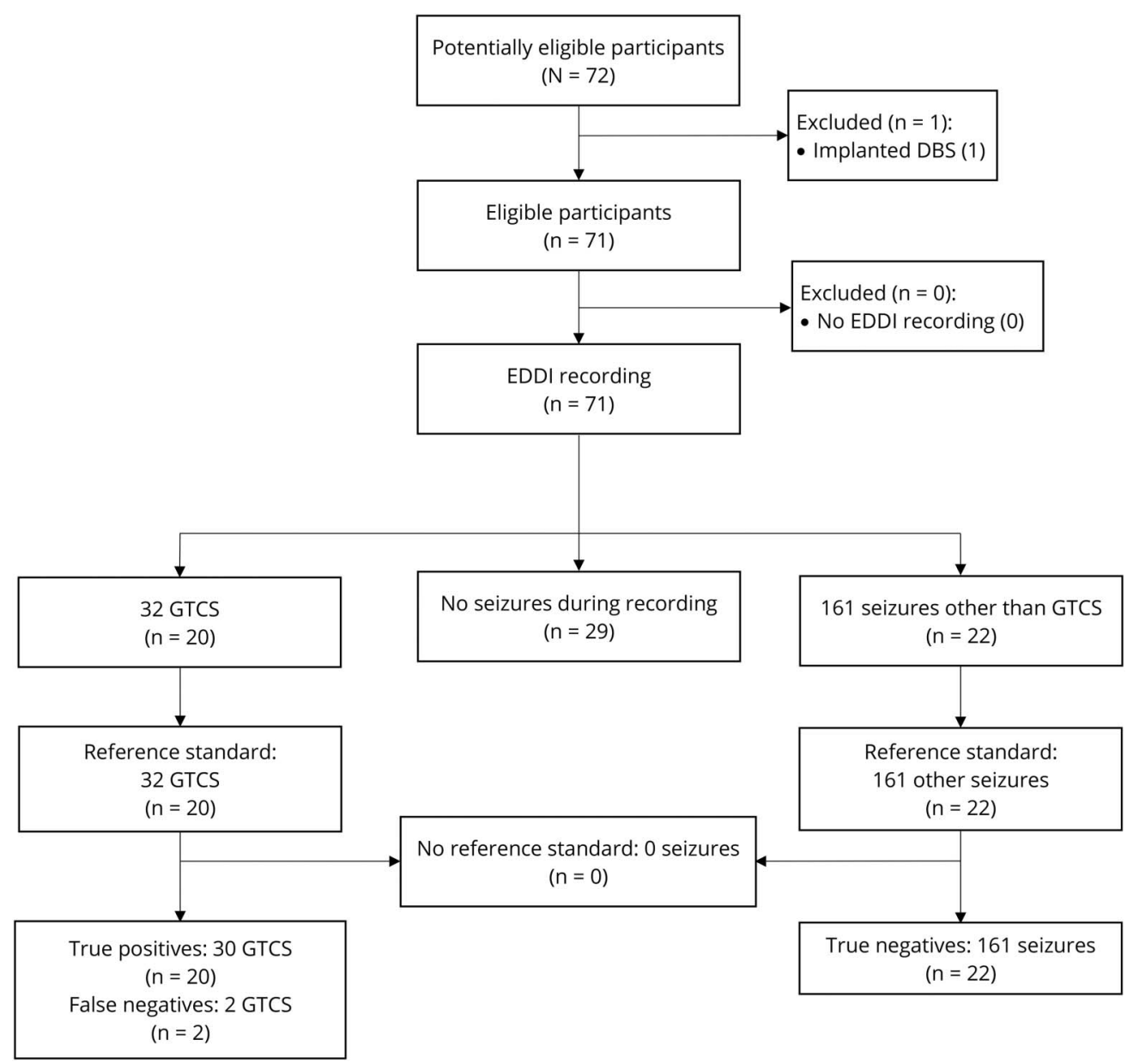

DBS = deep brain stimulation; EDDI = Epileptic seizure Detector Developed by IctalCare; GTCS = generalized tonic-clonic seizure.

The false discovery rate was $0.02 / \mathrm{h}$ (i.e., $0.67 / \mathrm{d}$ ). Twothirds of the patients $(47 / 71[66 \%])$ did not have false alarms. In 3 patients, the number of false alarms was $\geq 10$. The most common reason for false alarms was physiologic muscle activation: physical exercise (68\% of false alarms). The second most common reason was device malfunction: induction artifacts (18\%) and detaching electrodes (2.5\%). The vast majority of false alarms occurred during daytime. Only 2 nocturnal false alarms occurred: one was triggered by an episode classified as paroxysmal arousal, and the other one was triggered by stretching the arms during an arousal.

The ratio between false alarms and true alarms was $\leq 1$ for 17 of the 20 patients with GTCS (85\%), and in 13 patients with GTCS (65\%) the ratio was zero (i.e., no false alarms recorded). The range of the ratio was between 0 and 14 (median 0; mean 1.3). A total of 161 seizures other than GTCS occurred during the recording: 59 simple partial seizures (11 patients), 55 complex partial seizures (14 patients), 26 myoclonic jerks ( 5 patients), 6 absences (2 patients), 1 nonconvulsive status epilepticus, 14 convulsive psychogenic nonepileptic seizures (5 patients). None of these triggered seizure alarms.

\section{Discussion}

In this prospective, multicenter, blinded study on the accuracy of real-time detection of GTCS using an EMG-based wearable device (EDDI), we found that it had high sensitivity $(93.8 \%)$ and short detection latency ( 9 seconds). Specificity of the device was reflected by the low number of false alarms $(0.67 / d)$. None of 161 seizures other than GTCS triggered an alarm.

As the study was blinded, the alarm function of the device was turned off in this study. Nevertheless, the short detection latency suggests that timely warning is possible using this device. Large field studies, with long-term, ambulatory use of the device, are necessary to evaluate its potential in reducing the number of seizure-related injuries and ultimately the number of SUDEP.

Both undetected seizures occurred shortly after a detected GTCS. A possible explanation is that the mechanism of 
muscle activation during the tonic phase of recurring GTCS is different (smaller amplitude, shorter duration). Multiple GTCS occurred in 7 other patients in whom all GTCS were detected. Recurring seizures (i.e., with short interseizure period) occurred in the group of detected seizures too. Two of the detected, recurring seizures had interseizure periods (73 and 74 minutes) within the range of the interseizure periods of the undetected seizures (19-98 minutes). Thus, in at least some patients the second GTCS might remain undetected, when the interseizure period is short.

Surveys of large numbers of patients and their caregivers addressed the users' requirements for wearable seizure detection devices. ${ }^{7-10}$ Patients did not want to use implanted and EEGbased devices. Patch electrodes at invisible body sites were considered acceptable. ${ }^{7,8,10}$ Detection of seizures involving heavy movement and falls was considered most important by patients and caregivers, while for physicians, SUDEP prevention was the most significant goal. ${ }^{8}$ This emphasizes the importance of detecting GTCS, the seizure type that is targeted by our device. The most important feature for users was sensitivity, with most users requiring sensitivity over $90 \%$. Our device provides this. The required detection latency was under 10 seconds ${ }^{8}$ or 30 seconds. ${ }^{9}$ The median detection latency in our study was 9 seconds. In 2 cases, the muscle activation triggering the alarm occurred 3-4 seconds before any movement was observable in the video-EEG recordings, giving negative values for detection latency. Concerning the FAR, the results of the surveys varied broadly, but in all surveys users agreed that sensitivity is more important than FAR. In one of the surveys, the acceptable FAR was considered less than $1 /$ wk or up to 1 false alarm/detected seizure. ${ }^{8}$ Our device fulfilled the latter requirement, but the overall FAR was more than $1 / \mathrm{wk}(4.7 / \mathrm{wk})$. However, throughout the study, only 2 false alarms occurred during sleep, which is important for the quality of life of patients and caregivers.

The baseline frequency of GTCS prior to admission to the EMU was lower than what we recorded in the EMU. This means that in ambulatory use, the ratio between false alarms and true alarms would be higher than what we calculated in the EMU, and FAR may need to be improved for ambulatory use in medicated patients. Nevertheless, the baseline seizure frequency of GTCS was derived from the patients' seizure logs, and previous studies showed that many GTCS remain undetected by the patients. ${ }^{11}$

Device deficiency time was $<5 \%$. The device gives alarms when the electrode impedance is too high, so that the users can correct this. However, due to the blinding, this feature was disabled throughout the study. Thus, it is reasonable to assume that device deficiency time would be shorter when users are alerted about this.

Two different algorithms for unimodal EMG-based seizure detection have been developed by 2 groups. Both algorithms have been tested retrospectively, by offline analysis of data recorded with conventional amplifiers. ${ }^{17,18}$ Our algorithm is generic and targets the initial phase of GTCS. ${ }^{17}$ The retrospective offline analysis gave a sensitivity of $100 \%$. A modified version of this algorithm was implemented in the wearable device we have tested. In this prospective study, based on realtime seizure detection, 2 of the 32 seizures were missed. This demonstrates the importance of validating seizure detection devices using this design, rather than by offline, retrospective analysis. The other algorithm, developed by Brain Sentinel (San Antonio, TX), targets a later phase: the tonic-clonic interface. ${ }^{18}$ The algorithm is individualized and it utilizes the Hotelling $\mathrm{T}^{2}$ power analysis. The retrospective, offline analysis showed a sensitivity of 95\% (20 out of 21 GTCS from 11 patients were detected) and only 1 false alarm occurred. The average detection latency was within 20 seconds. ${ }^{18}$ Both algorithms have been registered for medical application: in Europe, EDDI obtained CE registration in 2013, and Brain Sentinel was granted de novo clearance by the Food and Drug Administration in 2017.

In spite of numerous attempts to develop noninvasive seizure detection, ${ }^{3,5}$ there are only 4 prospective studies on clinical validation of wearable devices using real-time seizure detection. ${ }^{22,25-27}$ All 4 studies used accelerometers. Two articles addressed the same device: SmartWatch. ${ }^{26,27}$ Although the pilot study showed promising results ( 7 out of 8 seizures were detected), ${ }^{26}$ in a large prospective study, only $31 \%$ of GTCS were detected. ${ }^{27}$ Another algorithm correctly identified 20 of 22 (91\%) seizures from 15 patients, and had a detection latency of 17 seconds. ${ }^{25}$ Although real-time, the analysis was done on a computer placed within 10 meters from the patient. A wearable accelerometer device (Epi-Care Free; Tunstall, Astoria, NY) detected GTCS with a sensitivity of $89.7 \%{ }^{22}$ Although the FAR was low $(0.2 / \mathrm{d})$, this was achieved by instructing patients to avoid movements that could trigger alarms with the arm where the sensor was placed. The detection latency was significantly longer (55 seconds) compared to EDDI. Accelerometers trigger the alarm in the later, clonic phase of the GTCS, while EDDI targets the initial part of the tonic phase, thus providing much earlier detection (9 seconds) of the seizure and the possibility of much earlier intervention.

The main limitation of our study is the intrinsic challenge of any validation study of wearable seizure detection devices: the testing was done in an EMU, which differs from the patients' home environment. This was necessary in order to have the reference standard. However, mobility of the patients was not restricted during monitoring, and they were encouraged to perform indoors physical activities similar to their habitual lifestyle. $^{21}$

Using the data recorded in this study, further optimization of the algorithm is planned, especially for decreasing the FAR. An alternative approach can be using individualized thresholds. It is noteworthy that the device was not triggered by any of the 14 convulsive psychogenic nonepileptic seizures that occurred during this study, considering that all 5 patients who 
had these were referred to the EMU on suspicion of GTCS. This raised the possibility of using the device for differential diagnostic purposes, as indicated previously. ${ }^{15}$

\section{Author contributions}

S.B., I.C., and P.W. contributed to the conception and design of the study. S.B., O.H., and M.F. acquired and analyzed data. I.C. monitored the logs from the detection device and provided technical support. S.B. drafted the manuscript and I.C. the figures. All authors contributed to editing the manuscript.

\section{Acknowledgment}

The authors thank the patients and their caregivers who participated in this study and the personnel of the epilepsy monitoring units of involved centers.

\section{Study funding}

This was an investigator-initiated study and no funding was granted to it. IctalCare, the manufacturer of the EDDI device, granted the investigators free-of-charge use of the device throughout the study period.

\section{Disclosure}

S. Beniczky reports no disclosures relevant to the manuscript. I. Conradsen was an employee of and is currently a consultant for IctalCare. She monitored the logs from the detection devices but did not have any influence on the logs with the time points generated by the real-time seizure detection of the device. She also gave technical assistance in form of training the personnel in the EMUs in using the device, but she was not present in the EMUs during the study and she was blinded to all clinical data, including the reference standard, prior to completion of the analyses. IctalCare, the manufacturer of the EDDI device, granted the investigators free-of-charge use of the device throughout the study period. O. Henning, M. Fabricius, and P. Wolf report no disclosures relevant to the manuscript. Go to Neurology.org/N for full disclosures.

Received July 3, 2017. Accepted in final form October 9, 2017.

\section{References}

1. Devinsky O, Hesdorffer DC, Thurman DJ, et al. Sudden unexpected death in epilepsy: epidemiology, mechanisms, and prevention. Lancet Neurol 2016;15:1075-1088.
2. Tomson T, Surges R, Delamont R, et al. Who to target in sudden unexpected death in epilepsy prevention and how? Risk factors, biomarkers, and intervention study designs. Epilepsia 2016;57(suppl 1):4-16.

3. Van de Vel A, Cuppens K, Bonroy B, et al. Non-EEG seizure detection systems and potential SUDEP prevention: state of the art: review and update. Seizure 2016;41: 141-153.

4. Brown S, Hanna J, Hirst J, et al. Statement of research need: the epilepsy deaths register: making every epilepsy death count. Available at: sudep.org/statementresearch-need. Accessed June 1, 2017.

5. Jory C, Shankar R, Coker D, et al. Safe and sound? A systematic literature review of seizure detection methods for personal use. Seizure 2016;36:4-15.

6. Schulze-Bonhage A, Sales F, Wagner K, et al. Views of patients with epilepsy on seizure prediction devices. Epilepsy Behav 2010;18:388-396.

7. Hoppe C, Feldmann M, Blachut B, et al. Novel techniques for automated seizure registration: patients' wants and needs. Epilepsy Behav 2015;52:1-7.

8. Van de Vel A, Smets K, Wouters K, Ceulemans B. Automated non-EEG based seizure detection: do users have a say? Epilepsy Behav 2016;62:121-128.

9. Tovar Quiroga DF, Britton JW, Wirrell EC. Patient and caregiver view on seizure detection devices: a survey study. Seizure 2016;41:179-181.

10. Patel AD, Moss R, Rust SW, et al. Patient-centered design criteria for wearable seizure detection devices. Epilepsy Behav 2016;64:116-121.

11. Blum DE, Eskola J, Bortz JJ, et al. Patient awareness of seizures. Neurology 1996;47: 260-264.

12. Beniczky S, Conradsen I, Pressler R, Wolf P. Quantitative analysis of surface electromyography: biomarkers for convulsive seizures. Clin Neurophysiol 2016;127: 2900-2907.

13. Conradsen I, Wolf $\mathrm{P}$, Sams T, et al. Patterns of muscle activation during generalized tonic and tonic-clonic epileptic seizures. Epilepsia 2011;52:2125-2132.

14. Beniczky S, Conradsen I, Moldovan M, et al. Quantitative analysis of surface electromyography during epileptic and nonepileptic convulsive seizures. Epilepsia 2014 55:1128-1134.

15. Beniczky S, Conradsen I, Moldovan M, et al. Automated differentiation between epileptic and non-epileptic convulsive seizures. Ann Neurol 2015;77:348-351.

16. Conradsen I, Moldovan M, Jennum P, et al. Dynamics of muscle activation during tonic-clonic seizures. Epilepsy Res 2012;104:84-93.

17. Conradsen I, Beniczky S, Hoppe K, et al. Automated algorithm for generalized tonicclonic epileptic seizure onset detection based on sEMG zero-crossing rate. IEEE Trans Biomed Eng 2012;59:579-585.

18. Szabó CÁ, Morgan LC, Karkar KM, et al. Electromyography-based seizure detector: preliminary results comparing a generalized tonic-clonic seizure detection algorithm to video-EEG recordings. Epilepsia 2015;56:1432-1437.

19. Conradsen I, Beniczky S, Wolf P, et al. Evaluation of novel algorithm embedded in a wearable sEMG device for seizure detection. Conf Proc IEEE Eng Med Biol Soc 2012;2012:2048-2051.

20. Bossuyt PM, Reitsma JB, Bruns DE, et al. STARD 2015: an updated list of essential items for reporting diagnostic accuracy studies. BMJ 2015;351:h5527.

21. Craciun L, Alving J, Gardella E, et al. Do patients need to be bedridden in the epilepsy monitoring unit? Safety data from a non-restrictive setting. Seizure 2017;49:13-16.

22. Beniczky S, Polster T, Kjaer TW, Hjalgrim H. Detection of generalized tonic-clonic seizures by a wireless wrist accelerometer: a prospective, multicenter study. Epilepsia 2013;54:e58-e61.

23. Jones SR, Carley S, Harrison M. An introduction to power and sample size estimation. Emerg Med J 2003;20:453-458.

24. Rheims S, Ryvlin P. Patients' safety in the epilepsy monitoring unit: time for revising practices. Curr Opin Neurol 2014;27:213-218.

25. Kramer U, Kipervasser S, Shlitner A, Kuzniecky R. A novel portable seizure detection alarm system: preliminary results. J Clin Neurophysiol 2011;28:36-38.

26. Lockman J, Fisher RS, Olson DM. Detection of seizure-like movements using a wrist accelerometer. Epilepsy Behav 2011;20:638-641.

27. Patterson AL, Mudigoudar B, Fulton S, et al. SmartWatch by SmartMonitor: assessment of seizure detection efficacy for various seizure types in children: a large prospective single-center study. Pediatr Neurol 2015;53:309-311. 


\section{Automated real-time detection of tonic-clonic seizures using a wearable EMG device} Sándor Beniczky, MD, PhD, Isa Conradsen, PhD, Oliver Henning, MD, Martin Fabricius, MD, DMSc,
and Peter Wolf, MD, DMSc

Cite as: Neurology ${ }^{\circledR}$ 2018;90:e428-e434. doi:10.1212/WNL.0000000000004893
Correspondence

Dr. Beniczky

sbz@filadelfia.dk

\section{Study question}

Can the Epileptic seizure Detector Developed by IctalCare (EDDI), a wearable EMG device, provide accurate automatic detection of generalized tonic-clonic seizures (GTCSs)?

\section{Summary answer}

The EDDI device can reliably detect GTCSs.

\section{What is known and what this article adds}

Wearable seizure detectors could alert caregivers to patients' potentially injury-inducing seizures and provide objective recording of seizure events. This study provides Class II evidence for the hypothesis that wearable EMGbased seizure detectors can provide accurate real-time GTCS detection.

\section{Participants and setting}

The study included 71 patients who had been referred to long-term video-EEG monitoring because of suspected GTCSs. The experiments were conducted in epilepsy monitoring units in Denmark and Norway between October 21, 2014, and January 23, 2017. The patients' median baseline GTCS frequency was 1.75 per month.

\section{Design, size, and duration}

For reference data, the patients underwent video-EEG monitoring. The EDDI device was unilaterally placed over a brachial biceps muscle and was set to silently record detected seizure events. The mean recording time per patient was 53.18 hours. The EDDI seizure-detection output data were read by evaluators blinded to the relevant patient's video-EEG data.

\section{Main results and the role of chance}

The video-EEG systems detected 32 GTCSs in 20 (28\%) patients. The EDDI device detected 30 of these GTCSs for a $93.8 \%$ sensitivity ( $95 \%$ confidence interval $86 \%-100 \%$ ). The 2 missed GTCSs were secondary seizures occurring



after detected GTCSs. The median detection latency was 9 seconds (range -4 to 48 seconds). The false alarm rate was 0.67 per day, but $47(66 \%)$ patients had no false alarms. Conversely, 3 (4\%) patients had $\geq 10$ false alarms. The video-EEG systems recorded 161 non-GTCS seizures, but the EDDI device did not record any of these.

\section{Bias, confounding, and other reasons for caution}

The experiment was conducted in epilepsy monitoring units, which may differ in important ways from the patients' home environments.

\section{Generalizability to other populations}

The study focused on detecting GTCSs, so it does not establish the EDDI device's utility for patients who experience other seizure types.

\section{Study funding/potential competing interests}

This study did not receive any funding. IctalCare granted freeof-charge use of the EDDI device. Dr. Conradsen is a former employee of and current consultant for IctalCare. Go to Neurology.org/ $\mathrm{N}$ for full disclosures. 


\section{Neurology}

Automated real-time detection of tonic-clonic seizures using a wearable EMG device

Sándor Beniczky, Isa Conradsen, Oliver Henning, et al.

Neurology 2018;90;e428-e434 Published Online before print January 5, 2018

DOI 10.1212/WNL.0000000000004893

This information is current as of January 5, 2018

Neurology ${ }^{\circledR}$ is the official journal of the American Academy of Neurology. Published continuously since 1951, it is now a weekly with 48 issues per year. Copyright $@ 2018$ The Author(s). Published by Wolters Kluwer Health, Inc. on behalf of the American Academy of Neurology.. All rights reserved. Print ISSN: 0028-3878. Online ISSN: 1526-632X.






\section{Updated Information \& Services}

\section{Supplementary Material}

\section{References}

Citations

Subspecialty Collections

Permissions \& Licensing

\section{Reprints}

including high resolution figures, can be found at:

http://n.neurology.org/content/90/5/e428.full

Supplementary material can be found at: http://n.neurology.org/content/suppl/2018/01/05/WNL.0000000000004 893.DC1

http://n.neurology.org/content/suppl/2019/03/07/WNL.0000000000004 893.DC2

This article cites 26 articles, 3 of which you can access for free at: http://n.neurology.org/content/90/5/e428.full\#ref-list-1

This article has been cited by 4 HighWire-hosted articles: http://n.neurology.org/content/90/5/e428.full\#\#otherarticles

This article, along with others on similar topics, appears in the following collection(s):

\section{All Clinical trials}

http://n.neurology.org/cgi/collection/all_clinical_trials

\section{Class II}

http://n.neurology.org/cgi/collection/class_ii

EMG

http://n.neurology.org/cgi/collection/emg

Epilepsy monitoring

http://n.neurology.org/cgi/collection/epilepsy_monitoring_

Generalized seizures

http://n.neurology.org/cgi/collection/generalized_seizures

Information about reproducing this article in parts (figures,tables) or in its entirety can be found online at:

http://www.neurology.org/about/about_the_journal\#permissions

Information about ordering reprints can be found online:

http://n.neurology.org/subscribers/advertise

Neurology ${ }^{\circledR}$ is the official journal of the American Academy of Neurology. Published continuously since 1951, it is now a weekly with 48 issues per year. Copyright @ 2018 The Author(s). Published by Wolters Kluwer Health, Inc. on behalf of the American Academy of Neurology.. All rights reserved. Print ISSN: 0028-3878. Online ISSN: 1526-632X.

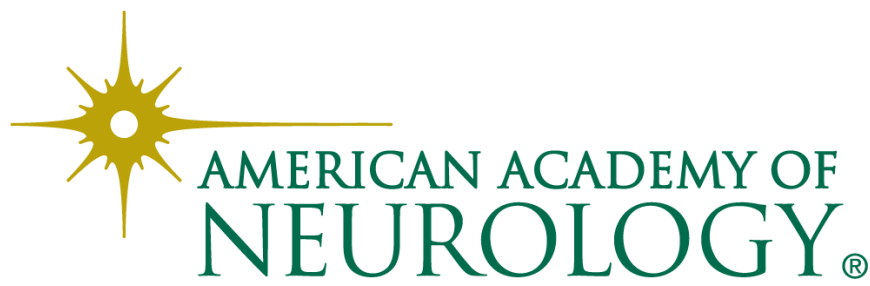

